\title{
Desenvolvimento folicular e desempenho produtivo no início do ciclo de postura em duas raças de poedeiras
}

\author{
Follicular development and productive performance at the onset of production in \\ two egg-laying breeds
}

\author{
Priscila Becker Ferreira' ${ }^{\mathrm{I}}$ André Favero ${ }^{\mathrm{II}}$ Alexandre Pires Rosa ${ }^{\mathrm{III}}$ André Macedo ${ }^{\mathrm{IV}}$ \\ Diogo Taschetto ${ }^{\mathrm{II}}$ José Guilherme Morschel Barbosa ${ }^{\mathrm{IV}}$
}

\section{RESUMO}

Avaliaram-se as características reprodutivas de duas raças puras de poedeiras semipesadas. Foram utilizadas 800 fêmeas, 400 da raça Plymouth Rock White (PRW) e 400 da raça Plymouth Rock Barred (PRB) no período compreendido entre a $16^{a}$ e a 24a semanas de idade, quando todas as aves tiveram o mesmo manejo e receberam água e ração ad libitum. Semanalmente, foram mensurados os parâmetros peso corporal, consumo alimentar, taxa de postura, conversão alimentar, peso dos ovos, peso de oviduto e número de folículos ovarianos. Foi utilizado um delineamento inteiramente casualizado (DIC) com dois tratamentos (PRW e $P R B)$ e cinco repetições de oitenta aves cada, sendo que os dados foram submetidos à análise de variância e as médias comparadas pelo teste " $T$ " de Student a 5\% de significância. Observou-se que na 24a semana de idade as aves da raça PRW apresentaram uma maior taxa de postura $(P=0,006)$ e melhor conversão alimentar $(P=0,020)$, comparado às aves da raça $P R B$. As aves $P R B$ apresentaram maior peso corporal, comparado ao peso das $P R W$, entretanto, o peso do oviduto em relação ao peso corporal na $24^{a}$ semana e o número de folículos grandes amarelos na $20^{\underline{a}}$ semana de idade foram maiores nas aves da raça PRW, comparado com as aves da raça $P R B(P=0,029)$. Aves poedeiras da raça $P R W$ são mais eficientes nos parâmetros produtivos e apresentam o desenvolvimento do aparelho reprodutivo mais precoce em relação às aves da $P R B$.

Palavras-chave: folículos, maturidade sexual, ovário, oviduto, ovos marrons.

\section{ABSTRACT}

It was evaluated the reproductive traits of two pure breed laying hens. There were used 800 females, 400 White
Plymouth Rock (PRW) and 400 Barred Plymouth Rock pullets, from 16 to 24 week of age. Pullets were managed in the same manner and received water and feed ad libitum. Body weight, feed intake, hen-day egg production, feed conversion, egg weight, oviduct weight and number of ovarian follicles were weekly measured. It was used a completely randomized design with two breeds (PRW and PRB) and five replicates of 80 pullets. The data were submitted to ANOVA and means compared by Student's $T$ test 5\% of significance. At 24 weeks of age the PRW pullets had a higher egg production $(P=0.006)$ and better feed conversion $(P=0.020)$ than the PRBs. The PRBs presented higher body weight than the PRWs, however, the number of large yellow follicles at 20 weeks, and the relative oviduct weight at 24 weeks of age were greater in the $P R W$ pullets $(P=0.029)$. The $P R W$ pullets were more efficient in the production parameters and were more precocious in the development of the reproductive tract than PRB pullets.

Key words: brown shell eggs, follicles, ovarian, oviduct, sexual maturity.

\section{INTRODUÇÃO}

Os programas de melhoramento genético, ao longo de décadas, selecionaram aves de postura mais produtivas, com menor peso corporal e baixo consumo de ração. No entanto, o aumento da produção de ovos vem chegando ao seu limite, e os pesquisadores já trabalham há algum tempo com outras características de importância econômica, como, por exemplo, a precocidade reprodutiva, que está diretamente relacionada ao número de ovos produzidos (ROMERO et al., 2009).

IPrograma de Pós-graduação em Zootecnia, Departamento de Zootecnia, Universidade Federal de Santa Maria (UFSM), Campus Universitário, 97105-900, Santa Maria, RS, Brasil. E-mail: pri_zoot@hotmail.com. Autor para correspondência.

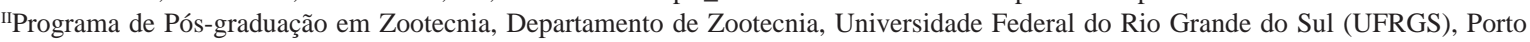
Alegre, RS, Brasil

IIIDepartamento de Zootecnia, UFSM, Santa Maria, RS, Brasil

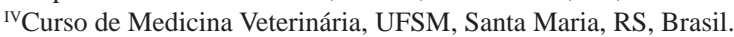


A aptidão reprodutiva de raças e linhagens de poedeiras pode ser avaliada por características como a idade ao primeiro ovo, morfologia e peso do ovário e do oviduto durante a maturação sexual e no período de postura. Essas características também podem ser observadas para avaliar a eficiência dos programas de manejo e alimentação, principalmente nas fases que antecedem a postura, servindo para identificar possíveis problemas, auxiliando na redução de custos de produção (SATTERLEE \& MARIN, 2004).

A puberdade não ocorre de maneira uniforme nas aves, sendo que vários fatores influenciam a produção do primeiro ovo. O ciclo ovulatório consiste no número de dias em que ocorre a oviposição, seguido por um dia de pausa. Assim, uma vez que os mecanismos de feedback entre ovário, pituitária e hipotálamo tenham alcançado a maturidade sexual, a ave é capaz de produzir ovos (ROBINSON et al., 1993). Os folículos destinados à ovulação estão organizados dentro de uma hierarquia que permite a ovulação diária e esta hierarquia é estabelecida pelos folículos pequenos (6 a 8mm). O folículo amarelo grande (FAG) que ultrapassar 8mm de diâmetro continua a se desenvolver e ovula (RUTZ et al., 2007).

Os folículos podem ser classificados em pelo menos quatro fases de desenvolvimento, que incluem os folículos de repouso, folículos brancos de crescimento lento, estágio de seleção do folículo e a diferenciação final. Em aves de postura, é esperado que apenas um folículo fosse liberado de cada vez. Dessa forma, os folículos amarelos grandes entram em hierarquia e o folículo de maior tamanho fica destinado a ovular primeiro (F1); o segundo maior pode ovular no dia seguinte (F2) e assim sucessivamente (JOHNSON, 1993).

Um número ideal de folículos amarelos grandes (FAG) pode estar associado com posturas de longa sequência, sendo de seis a oito FAG, um número normal para poedeiras de alta produção (WILLIAMS \& SHARP, 1978). Alguns fatores de manejo podem estimular uma produção e maturação excessiva de FAG, como programa de iluminação artificial mal conduzido, estresse térmico, manejo alimentar, entre outros. Esses fatores podem ser responsáveis por ocasionar perdas associadas à oviposição, como a produção de ovos de gema dupla, deformidades de casca, ovulação e postura interna (ROBINSON et al., 1993 e YU et al., 1992).

O programa de melhoramento genético do Laboratório de Avicultura da Universidade Federal de Santa Maria produz, desde 1988, poedeiras de ovos marrons das raças Plymouth Rock White (PRW) e Plymouth Rock Barred (PRB). Essas raças, quando cruzadas com galos geneticamente "gold" ou não barrados, geram pintinhos autosexáveis pela coloração da penugem. Por isso, aves PRW e PRB vêm sendo intensamente utilizadas como linha fêmea nos cruzamentos para produção de híbridos com alto potencial produtivo (FIGUEIREDO et al., 2003).

Assim, identificar as diferenças produtivas e reprodutivas destas raças permite calibrar normas de manejo e alimentação, de forma mais eficiente e diferenciada, levando em consideração a interação genótipo ambiente. Neste trabalho, objetivouse comparar a maturidade sexual através do desenvolvimento folicular e os índices produtivos destas duas raças no período que compreende da $16^{\mathrm{a}}$ a $24^{\text {a }}$ semana de idade.

\section{MATERIAL E MÉTODOS}

O estudo foi conduzido no Laboratório de Avicultura (LAVIC) do Departamento de Zootecnia da Universidade Federal de Santa Maria. Foram utilizadas 400 fêmeas da raça Plymouth Rock White (PRW) e 400 fêmeas da raça Plymouth Rock Barred (PRB), a partir de 16 semanas de idade. As aves foram alojadas em um aviário experimental de $300 \mathrm{~m}^{2}$, dotado de ventiladores e aspersores, dividido em 10 unidades experimentais de $15,75 \mathrm{~m}^{2}$ com 80 aves cada, criadas sobre cama de maravalha, sendo cada boxe equipado com dois comedouros tubulares, um bebedouro pendular e oito ninhos para postura de $40 \mathrm{~cm}^{2}$ cada, com cama de maravalha para evitar a quebra dos ovos.

O período experimental compreendeu da $16^{\text {a }}$ a $24^{\text {a }}$ semana de idade e, durante esse período, todas as aves tiveram o mesmo manejo. A água e a ração foram fornecidas ad libitum, sendo que todas as aves receberam a mesma dieta, a qual foi formulada à base de milho, farelo de soja e farelo de trigo, atendendo as exigências nutricionais conforme a fase de produção. As fases foram divididas em: inicial da 1a a $6^{\text {a }}$ semana de idade, recria da 7a a $18^{-a}$ semana de idade, pré-postura da $19^{\underline{a}}$ a $21^{\text {a }}$ semana de idade e postura da $22^{\text {a }}$ a $40^{\text {a }}$ semana de idade. Os níveis nutricionais foram de 22,08\%, 14,42\%, 15,51\% e $16,00 \%$ de proteína; $2.820,2.879,2.773$ e $2.850 \mathrm{Kcal}$ $\mathrm{kg}^{-1}$ de energia metabolizável; 0,96\%, 0,80\%, 1,46\% e $4,20 \%$ de cálcio; $0,50 \%, 0,31 \%, 0,45 \%$ e $0,39 \%$ de fósforo disponível, para as fases inicial, recria, pré-postura e postura, respectivamente, conforme as recomendações de ROSTAGNO et al. (2000). 
As poedeiras receberam uma iluminação artificial constante de 14 horas de luz por dia até 18 semanas de idade e, a partir da 18 ${ }^{\mathrm{a}}$ semana, foram acrescidos 30 minutos por semana de iluminação artificial, até atingir o limite de 17 horas de luminosidade $\mathrm{dia}^{-1}$. A postura dos ovos iniciou na $20^{\mathrm{a}}$ semana de idade e as coletas de ovos foram realizadas quatro vezes ao dia.

Semanalmente, foram mensurados os parâmetros de peso corporal, consumo de ração, taxa de postura, conversão alimentar, peso dos ovos, peso de oviduto e número de folículos ovarianos. Para determinação de peso corporal, consumo de ração e conversão alimentar, todas as aves foram pesadas semanalmente, assim como a ração fornecida e as sobras. O peso dos ovos foi determinado através da pesagem semanal de todos os ovos produzidos em um dia. Os pesos de ovário, oviduto e o número de folículos ovarianos foram obtidos semanalmente através do abate de três aves por repetição, selecionadas ao acaso. As aves foram pesadas individualmente, abatidas por deslocamento cervical, conforme as normas de bem estar animal, e realizada a coleta de oviduto e ovário. Os órgãos coletados foram pesados e realizou-se a contagem dos folículos ovarianos com o auxilio de um disco plástico com orifícios de 10, 5 e 3mm de diâmetro. Conforme o tamanho de cada folículo, eles foram classificados como: folículo amarelo grande (FAG), os folículos com diâmetro maior que 10mm; folículo amarelo pequeno (FAP), os folículos com diâmetro entre 5 e 10mm; e folículo branco grande (FBG), os folículos com diâmetro entre 3 a $5 \mathrm{~mm}$.

O delineamento experimental foi 0 inteiramente casualizado (DIC) com dois tratamentos (PRW e PRB) e cinco repetições de oitenta aves cada. Para os parâmetros de peso de oviduto, peso de ovário e número de folículos ovarianos, cada repetição foi composta por três aves. Os dados foram submetidos à análise de variância e as médias comparadas pelo teste " $T$ " de Student, a 5\% de significância. As análises estatísticas foram realizadas pelo programa estatístico SAS $^{\circledR} \quad$ versão 9.2 (STATISTICAL ANALYSIS SYSTEM, 2001).

\section{RESULTADOS E DISCUSSÃO}

Durante todo o período experimental, houve diferença significativa entre o peso corporal das aves das diferentes raças, sendo as aves da raça Plymouth Rock Barred (PRB) mais pesadas que as aves Plymouth Rock White (PRW), conforme tabela 1. O incremento no peso corporal das aves, de um modo geral, é mais acentuado e evidente nas semanas próximas ao pico da produção, comportando-se de maneira regular e constante posteriormente a este período. As aves das duas raças diferiram significativamente no consumo alimentar na maioria dos períodos observados (Tabela 1). As aves

Tabela 1 - Peso corporal e consumo de ração de fêmeas Plymouth Rock White (PRW) e Plymouth Rock Barred (PRB) da $16^{a}$ a $24^{\underline{a}}$ semana de idade.

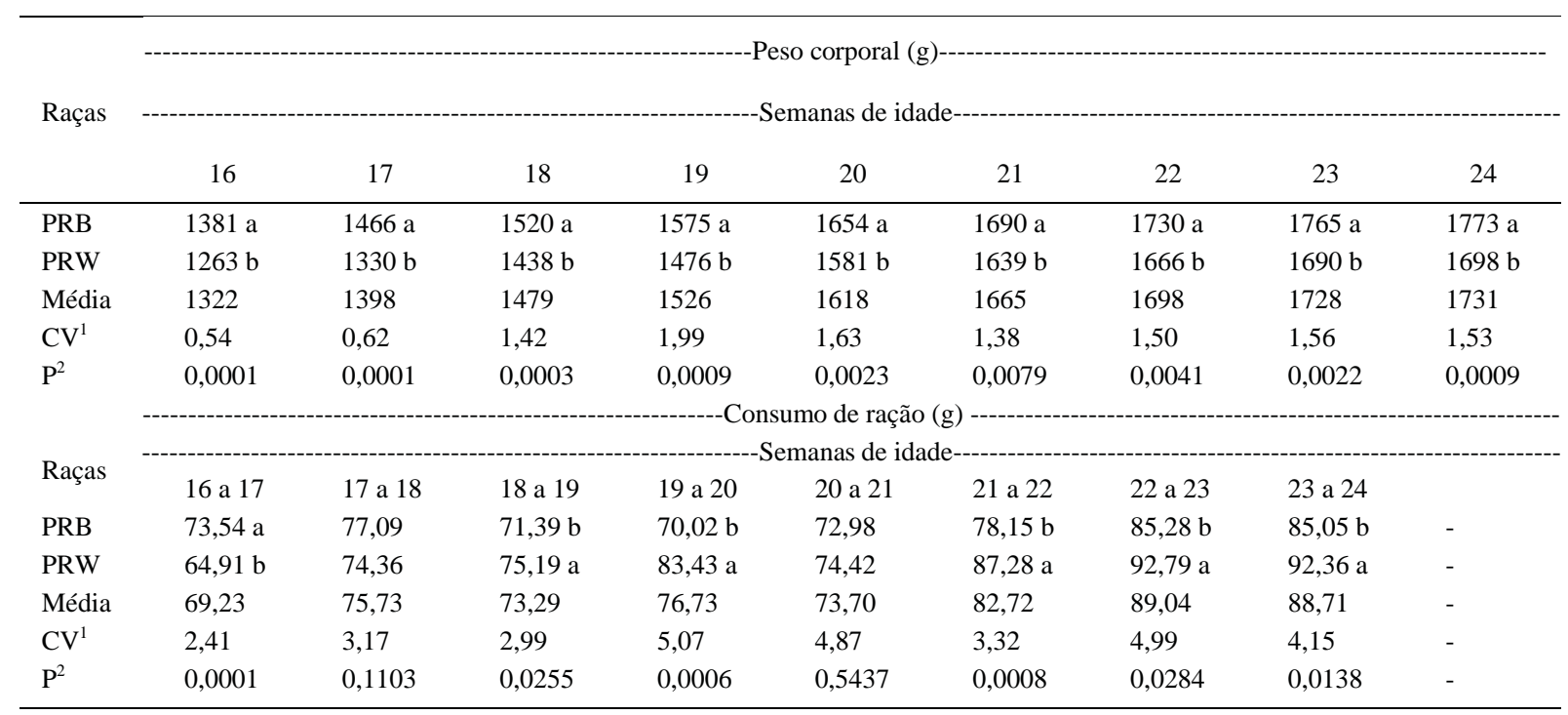

a, b Médias seguidas por letras diferentes nas colunas diferem entre si pelo Teste "T" de Student.

${ }^{1}$ Coeficiente de variação (\%).

${ }^{2}$ Significância.

Ciência Rural, v.44, n.3, mar, 2014. 
PRB apresentaram consumo alimentar superior às aves PRW durante a $16^{\mathrm{a}}$ semana de idade $(\mathrm{P}=0,0001)$. A partir da 18 a semana de idade, as aves PRW apresentaram consumo de ração superior em relação às aves PRB, porém, na 20aㅡ semana de idade, essa diferença não foi significativa.

Diferenças significativas quanto à produtividade das raças PRB e PRW foram encontradas durante a 24- semana de idade, quando as aves PRW apresentaram a maior taxa de postura ( $\mathrm{P}=0,0064)$, conforme tabela 2 . As aves PRW tiveram maior consumo alimentar que as aves PRB, podendo ser atribuído esse fato pelo aspecto produtivo, pois produziram mais ovos e consequentemente obtiveram uma maior demanda nutricional. A conversão alimentar das aves PRB foi de 1,60kg dúzia-1 de ovos, apresentando-se pior em relação à conversão das aves PRW, que foi de $1,28 \mathrm{~kg}_{\text {dúzia }}{ }^{-1}$ de ovos durante a $24^{\mathrm{a}}$ semana, sendo atribuída a maior taxa de postura das aves PRW (Tabela 2). Esses resultados sugerem a realização de manejos alimentares e/ou nutricionais diferentes para as duas raças, como, por exemplo, o aumento dos níveis nutricionais para a raça PRW, diminuindo o consumo de ração. Também pode ser realizado o controle de ração para as aves PRB, para evitar um alto peso corporal ou o acúmulo de gordura, o que afeta negativamente a produção de ovos.

Os pesos dos ovos das diferentes raças tiveram diferença significativa na 22aㅗ $23^{\underline{a}}$ e $24^{\text {a }}$ semana de idade. As aves PRB apresentaram peso médio de ovo inferior às aves PRW, conforme tabela 2. O peso médio dos ovos tende a aumentar proporcionalmente, à medida que o peso corporal das aves também aumenta, porém, neste trabalho, as aves PRW apresentaram maior peso médio de ovos e menor peso corporal em relação às aves PRB (da 22a a 24a semana de idade). ROBINSON et al. (2001), trabalhando com as linhagens Isa Babcock (precoces) e Shaver White (tardias), não observaram diferenças significativas em relação ao peso dos ovos.

O peso de ovário e a percentagem do seu peso em relação ao peso corporal das aves não apresentaram diferenças significativas entre as raças estudadas durante o período experimental, conforme mostra a tabela 3. Segundo SATTERLEE \& MARIN (2004), os ovários, no período pré-puberal, pesam cerca de $0,20 \mathrm{~g}$ e podem chegar a ter $50 \mathrm{~g}$. Durante o período experimental deste trabalho, pôde-se observar esse desenvolvimento progressivo do ovário das aves PRB e PRW, as quais atingiram, na

Tabela 2 - Taxa de postura (\%), conversão alimentar ( $\mathrm{kg} \mathrm{dz}^{-1}$ ) e peso médio de ovos (g) de fêmeas Plymouth Rock White (PRW) e Plymouth Rock Barred (PRB) da 20a a 24 semana de idade.

\begin{tabular}{|c|c|c|c|c|c|}
\hline Raças & 20 & 21 & 22 & 23 & 24 \\
\hline PRB & 1,63 & 10,11 & 29,65 & 39,29 & $48,42 \mathrm{~b}$ \\
\hline PRW & 1,45 & 10,15 & 36,88 & 49,51 & $65,64 \mathrm{a}$ \\
\hline Média & 1,54 & 10,13 & 33,27 & 44,40 & 57,03 \\
\hline $\mathrm{CV}^{1}$ & 6,44 & 3,73 & 1,87 & 2,05 & 1,30 \\
\hline $\mathrm{P}^{2}$ & 0,7802 & 0,9882 & 0,1031 & 0,1131 & 0,0064 \\
\hline PRB & 104,89 & 7,55 & $\begin{array}{l}\text { aliment } \\
2,53\end{array}$ & 2,02 & $1,60 \mathrm{a}$ \\
\hline PRW & 84,61 & 8,13 & 2,24 & 1,78 & $1,28 \mathrm{~b}$ \\
\hline Média & 94,75 & 7,84 & 2,38 & 1,90 & 1,44 \\
\hline $\mathrm{CV}^{1}$ & 5,26 & 4,60 & 2,61 & 1,98 & 1,22 \\
\hline $\mathrm{P}^{2}$ & 0,7808 & 0,806 & 0,3718 & 0,3418 & 0,0202 \\
\hline PRB & 35,57 & 37,83 & $39,88 \mathrm{~b}$ & $41,58 \mathrm{~b}$ & $42,62 \mathrm{~b}$ \\
\hline PRW & 38,17 & 41,27 & 41,53 a & 43,87 a & $45,00 \mathrm{a}$ \\
\hline Média & 36,87 & 39,55 & 40,71 & 42,73 & 43,81 \\
\hline $\mathrm{CV}^{1}$ & 10,99 & 6,31 & 2,67 & 2,61 & 2,89 \\
\hline $\mathrm{P}^{2}$ & 0,3388 & 0,061 & 0,0436 & 0,0029 & 0,0176 \\
\hline
\end{tabular}

\footnotetext{
${ }^{\mathrm{a}, \mathrm{b}}$ Médias seguidas por letras diferentes nas colunas diferem entre si pelo Teste "T” de Student.

${ }^{1}$ Coeficiente de variação (\%).

${ }^{2}$ Significância.
} 
Tabela 3 - Peso de ovário (g), peso de oviduto (g), peso de ovário e peso de oviduto em relação ao peso corporal de fêmeas Plymouth Rock White (PRW) e Plymouth Rock Barred (PRB) da $16^{\underline{a}}$ a $24^{\underline{a}}$ semana de idade.

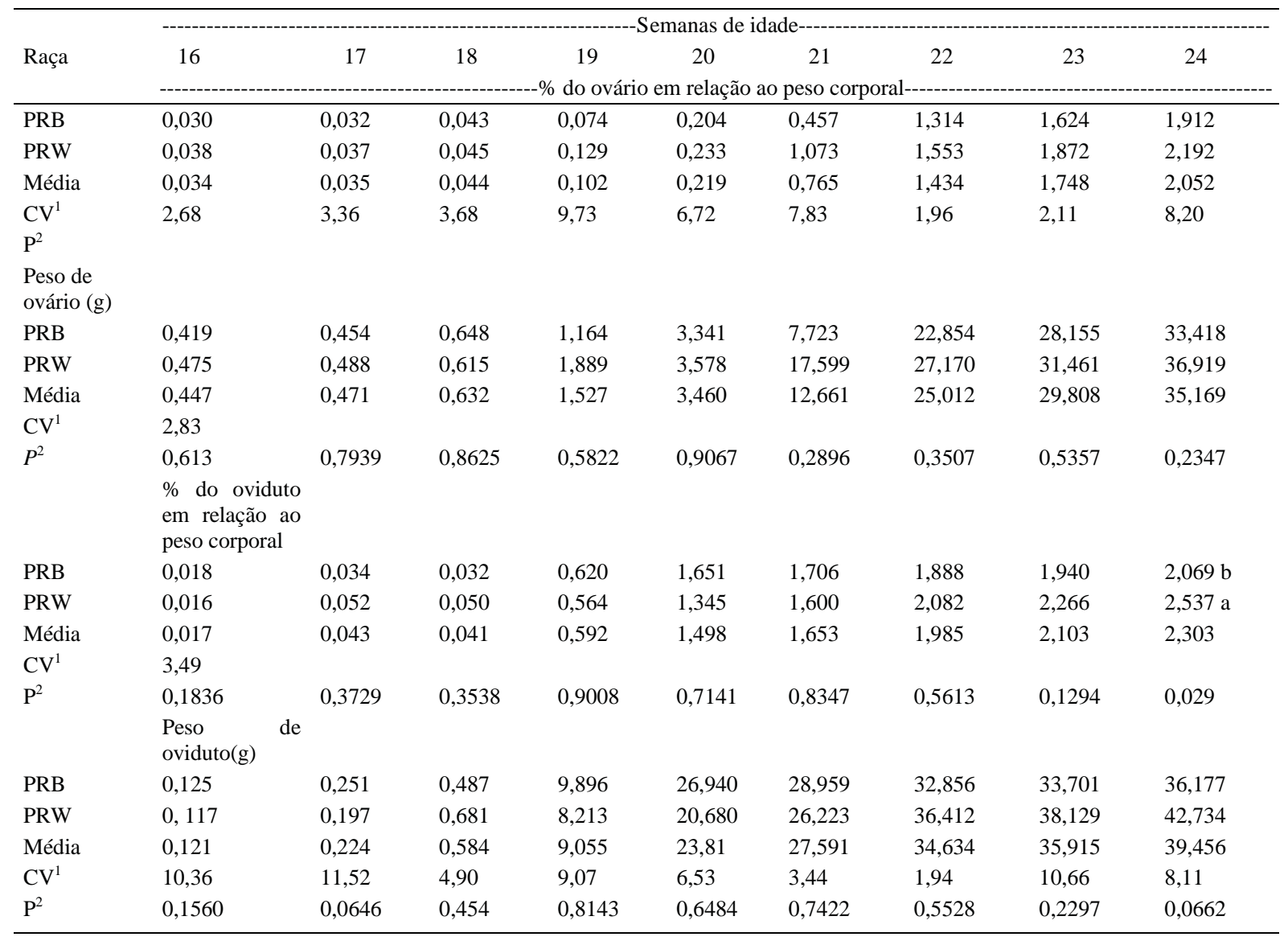

${ }^{\mathrm{a}, \mathrm{b}}$ Médias seguidas por letras diferentes nas colunas diferem entre si pelo Teste “T” de Student.

${ }^{1}$ Coeficiente de variação (\%).

${ }^{2}$ Significância.

24a semana de idade, peso de ovário igual a 33,42g e $36,91 \mathrm{~g}$, respectivamente.

Os resultados deste trabalho diferem dos encontrados por ROBINSON et al. (2001), que, trabalhando com diferentes linhagens, observaram maior peso de ovário na linhagem precoce, as 17, 21 e 22 semanas de idade. Entretanto, RENEMA et al. (2001) não encontraram diferença significativa no peso médio de ovário, comparando linhagens tradicionais e modernas (Shaver Starcross 288), mas observaram diferença significativa no peso de ovário em relação ao peso corporal.

O peso de oviduto apresentou diferença significativa na 24- semana de idade, quando aves PRW tiveram maior peso de oviduto em relação ao peso corporal, diferença essa não encontrada em relação ao peso de oviduto em gramas (Tabela 3). ROBINSON et al. (2001), em trabalho realizado com poedeiras, encontrou peso de oviduto superior em linhagem de aves da raça Isa Babcock, em comparação a aves da raça Shaver White, durante a 19a, $21^{\underline{a}}$ e 22a semana de idade. Ao estudar a morfologia reprodutiva de aves tradicionais e modernas oriundas da raça Leghorn, RENEMA et al. (2001) relatam que aves modernas (Shaver Starcross 288) apresentam ovidutos $10 \%$ maiores em relação a ovidutos de linhagens tradicionais.

A contagem de folículos amarelos grandes apresentou diferença significativa no início da fase produtiva das aves as 20 semanas de idade (Tabela 4). Aves PRW apresentaram maior número de folículos ovarianos em relação às aves PRB. Isso mostra uma organização aprimorada e importante na hierarquia folicular nos ovários de aves PRW, devido à maior produção e maturação de células foliculares mais desenvolvidas e próximas a ovular, mostrando assim 
Tabela 4 - Número de folículos ovarianos mensurados da $20^{\mathrm{a}}$ a $24^{\mathrm{a}}$ semana de idade de Plymouth Rock White (PRW) e Plymouth Rock Barred (PRB).

\begin{tabular}{|c|c|c|c|c|c|}
\hline Raça & 20 & 21 & 22 & 23 & 24 \\
\hline PRB & $0,33 \mathrm{~b}$ & 4,00 & 4,67 & 4,67 & 5,33 \\
\hline PRW & $1,67 \mathrm{a}$ & 3,33 & 5,33 & 5,67 & 6,00 \\
\hline Média & 1,00 & 3,67 & 5,00 & 5,17 & 5,67 \\
\hline $\mathrm{CV}^{1}$ & 5,77 & 8,03 & 11,54 & 11,17 & 19,06 \\
\hline $\mathrm{P}^{2}$ & 0,0477 & 0,7953 & 0,2302 & 0,1012 & 0,4918 \\
\hline PRB & 5,67 & 4,67 & 7,33 & 9,33 & 11,67 \\
\hline PRW & 1,67 & 6,33 & 5,00 & 6,00 & 6,67 \\
\hline Média & 3,67 & 5,50 & 6,17 & 7,67 & 9,17 \\
\hline $\mathrm{CV}^{1}$ & 19,73 & 7,68 & 4,34 & 3,37 & 3,42 \\
\hline $\mathrm{P}^{2}$ & 0,5354 & 0,6541 & 0,3459 & 0,1890 & 0,1226 \\
\hline PRB & 12,00 & 33,33 & 34,33 & 35,33 a & 36,00 \\
\hline PRW & 7,33 & 25,00 & 26,00 & $26,33 \mathrm{~b}$ & 29,67 \\
\hline Média & 9,67 & 29,17 & 30,17 & 30,83 & 32,83 \\
\hline $\mathrm{CV}^{1}$ & 7,18 & 4,10 & 3,83 & 6,35 & 3,19 \\
\hline $\mathrm{P}^{2}$ & 0,4565 & 0,4417 & 0,4272 & 0,0049 & 0,5009 \\
\hline
\end{tabular}

${ }^{\text {a, b }}$ Médias seguidas por letras diferentes nas colunas diferem entre si pelo Teste " $T$ ” de Student.

* Folículo amarelo grande: > 10mm de diâmetro.

** Folículo amarelo pequeno: entre 5 e 10mm de diâmetro.

*** Folículo branco grande: de 3 a $5 \mathrm{~mm}$ de diâmetro.

${ }^{1}$ Coeficiente de variação (\%).

${ }^{2}$ Significância.

a precocidade de aves PRW em comparação às aves da raça PRB. Dessa maneira, as PRW produzem e sequenciam uma quantidade maior de folículos maduros que poderão ser ovulados posteriormente. Esse resultado vem ao encontro com a maior produção de ovos na 24로 semana de idade das aves da raça PRW em relação às aves da raça PRB.

Durante o período de avaliação, o número de folículos amarelos pequeno não obteve diferença significativa conforme tabela 4. ROBINSON et al. (2001), trabalhando com poedeiras durante as 19, 21, 22 e 23 semanas de idade encontraram maior número de folículos FAG e FAP em aves da raça Isa Babcock, em relação às aves da raça Shaver White.

Na contagem de folículos grandes brancos (FBG), na 23aㅗ semana de idade, verificou-se diferença ( $\mathrm{P}=0,0049)$, já que as aves $\mathrm{PRB}$ apresentaram maior quantia desses folículos que as aves da raça PRW (Tabela 4). RENEMA et al. (2001) também verificaram que aves da raça Leghorn tradicionais (não selecionadas) apresentam um maior número de folículos pós-ovulatórios em relação às aves de linhagens modernas.

\section{CONCLUSÃO}

Aves poedeiras da raça Plymouth Rock White são mais eficientes nos parâmetros produtivos e apresentam o desenvolvimento do aparelho reprodutivo mais precoce em relação às aves da raça Plymouth Rock Barred.

\section{REFERÊNCIAS}

FIGUEIREDO, E.A.P. et al. Raças e linhagens de galinhas para criações comerciais e alternativas no Brasil. Concórdia SC: Ministério da Agricultura, Pecuária e Abastecimento, 2003. (Comunicado técnico). ISSN 0100-8862.

JOHNSON, A.L. Regulation of follicle differentiation by gonadotropins and growth factors. Poultry Science, v.72, p.867873, 1993. Disponível em: <http://ps.fass.org/search?fulltext=Reg ulation+of+follicle+differentiation+by+gonadotropins+and+grow th+factors\&submit=yes\&x=18\&y=4>. Acesso em: 13 dez. 2011. doi: $10.3382 / p s .0720867$.

RENEMA, R.A. et al. The effects of genetic strain and feeding regimen on carcass morphology traits at sexual maturity. Poultry Science, v.80, p.47-56, 2001. Disponível em: <http://ps.fass.org/ content/80/1/47.full.pdf+html>. Acesso em: 24 jan. 2012. 
ROBINSON, F.E. et al. Carcass traits, ovarian morphology and egg laying characteristics in early versus late maturing strains of commercial egg-type hens. Poultry Science, v.80, p.37-46, 2001. Disponível em: <http://ps.fass.org/content/80/1/37.full.pdf+html> . Acesso em: 14 jan. 2012.

ROBINSON, F.E. et al. The relationship between body weight and reproductive efficiency in meat-type chickens. Poultry Science, v.72, p.912-922, 1993. Disponível em: <http://ps.fass.org/ content/72/5/912.full.pdf+html>. Acesso em: 24 jan. 2012. doi: 10.3382/ps.0720912.

ROMERO, R.F. et al. Effect of reducing body weight variability on the sexual maturation and reproductive performance of broiler breeder females. Poultry Science, v.88, p.445-452, 2009. Disponível em: <http://ps.fass.org/content/88/2/445.full. pdf + html?sid=11ed96d5-46e7-469f-821c-f493ccf6d195>. Acesso em: 26 jan. 2012. doi: 10.3382/ps.2008-00165.

ROSTAGNO, H.S. et al. Tabelas brasileiras para suínos e aves: composição de alimentos e exigências nutricionais. Viçosa, MG: UFV, 2000. p. 186.

RUTZ, F. et al. Avanços na fisiologia e desempenho reprodutivo de aves. Revista Brasileira Reprodução Animal, v.31, n.3, p.307-317, 2007. Disponível em: <http://www.cbra.org.br/pages/ publicacoes/rbra/download/307.pdf>. Acesso em: 10 jan. 2012.

SATTERLEE, D.G.; MARIN, R.H. Pubic spread development and first egg lay in japanese quail. Journal of Applied Poultry Research, v.13, p.207-212, 2004.

SAS (STATISTICAL ANALYSES SYSTEM). SAS/STAT(r) 9.2 User's guide. Cary, 2001. 7857p.

WILLIAMS, J.B.; SHARP, P.J. Ovarian morphology and rates of ovarian follicular development in laying broiler breeders and commercial egg producing hens. British Poultry Science, v.19, p.387-395, 1978. Disponível em: <http://www.tandfonline.com/ doi/abs/10.1080/00071667808416490>. Acesso em: 14 dez. 2011. doi: 10.1080/00071667808416490.

YU, M.W. et al. Effect of feed allowance during rearing and breeding on female broiler breeders. 2. Ovarian morphology and production. Poultry Science, v.71, p.1750-1761, 1992. Disponível em: <http://ps.fass.org/content/71/10/1750.full. pdf+html?sid=07aa9e2a-3f81-4615-99c4-8baf3a961e82>. Acesso em: 25 jan. 2012. doi: 10.3382/ps.0711750. 